\title{
ANTENATAL VACCINATION AGAINST INFLUENZA AND PERTUSSIS IN GREECE
}

M. Zorbaㄹ D. Gkentzi², E. Papachatzi², M. Marangos ${ }^{3}$, A. Karatza², G. Dimitriou²

1 University of Patras, Department of Public Health, Patras, Greece.

2University General Hospital of Patras, Department of Paediatrics, Patras, Greece.

${ }^{3}$ University General Hospital of Patras, Department of Infectious Diseases, Patras, Greece.

\section{BACKGROUND AND AIMS}

- Bordetella pertussis causes serious illness among neonates and infants. Infants under 6 months of age have the highest rate of infection, compared to other ages, and $\geq 90 \%$ of pertussis deaths occur in infants under 3 months of age (1).

- Strategies to prevent pertussis in this population include a booster maternal immunization during third trimester of pregnancy (transplacental antibodies transmission) and targeted immunization ("cocooning") of infant caregivers (2).

- Neonates, having an immature immune system, are in high risk for influenza infection, with clinical manifestations that vary from mild symptoms (respiratory tract) to severe illness, with lethal outcome(3).

- In Greece, the National Immunization Schedule, recommends that pregnant women should receive influenza vaccination, at any time during pregnancy and Tdap vaccine at 27 - 36 weeks of gestation.

The main purpose of our study was to assess the knowledge, attitudes, beliefs, and factors associated with the antenatal vaccine uptake of pregnant women

\section{MATERIALS AND METHODS}

- A prospective cross-sectional study was conducted between January and June 2017 in six hospitals of Western Greece.

- The study population consisted of pregnant women who attended obstetric outpatient clinics and inpatient women, who gave birth in the postnatal ward.

- The study was conducted using a closed-ended questionnaire of 28 questions on demographics, knowledge and attitude towards antenatal vaccination.

\section{RESULTS}

- 432 women completed the questionnaire (response rate of $86.4 \%$ ), 324 (75\%) were pregnant , 108 (25\%) had just given birth .

-Although the majority of women were aware of both diseases $(66.9 \%, 289)$, they admitted lack of adequate information offered from all sources about antenatal vaccination $(73.4 \%, 317)$.

- There was poor awareness that the vaccination is safe during pregnancy $(95,22 \%)$.

\section{RESULTS (continued)}

- Few women even believed that maternal vaccination can cause birth defects $(26,5 \%)$ and autism $(13,2.5 \%)$.

- Only $76(17.6 \%)$ of the study participants were aware that all pregnant women should receive influenza and pertussis vaccines.

- The majority of women $(349,80.8 \%)$ did not receive any recommendations from their obstetrician who is their major antenatal care provider.

- Only 26 (6\%) of women had been offered the vaccines during current pregnancy.

- A binary logistic regression analysis was performed to determine the factors that influence antenatal vaccine uptake taking into account the demographic characteristics, knowledge, and attitude regarding vaccine uptake.

- We found significant impact to vaccine uptake $(\theta=1.704, p=0.000)$, when participants had prior vaccine uptake, were aware of the National Immunization Schedule recommendation $(B=1.286, p=0.006)$ and obstetrician 's recommendation $(B=0.962$, $p=0.049)$.

\section{CONCLUSIONS}

- The knowledge and uptake of influenza and pertussis vaccines among pregnant women in Greece was found to be poor and significantly lower, compared to other European countries and the USA.

- There is substantial room for improvement among antenatal care providers in both patient education and offering the vaccine.

\section{REFERENCES}

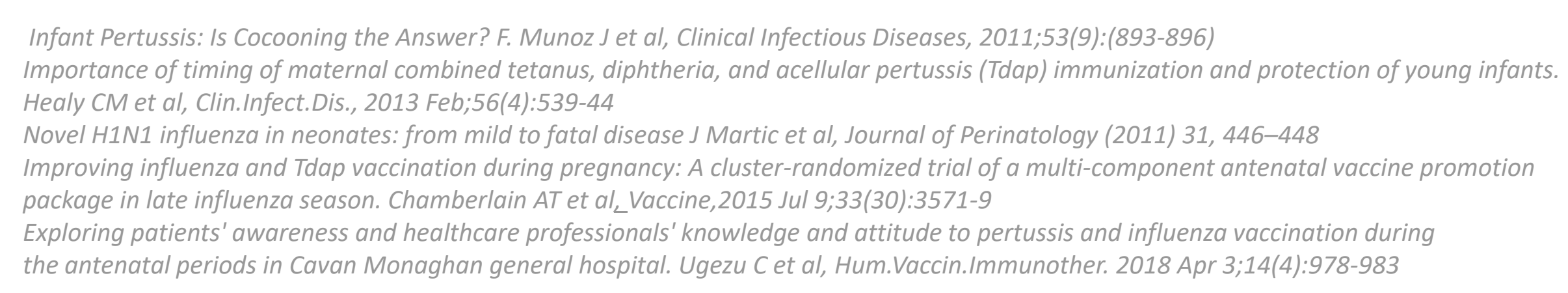

